\title{
Prediction of 3000-m Running Performance Using Classic Physiological Respiratory Responses
}

\author{
Thiago F. Lourenço ${ }^{1 *}$, Fernando O. C. da Silva ${ }^{1}$, Lucas S. Tessutti ${ }^{1}$, Carlos E. da Silva ${ }^{1}$, Cesar C. C. Abad ${ }^{2}$ \\ ${ }^{1}$ Biochemistry Department, State University of Campinas, Cidade Universitária Zeferino Vaz, Campinas, SP, 13083-970, Brazil \\ ${ }^{2}$ Department of Phyical Education, SENAC University, Av. Eng.Eusébio Stevaux São Paulo, SP 04696-000, Brazil
}

Corresponding Author: Thiago F. Lourenço, E-mail: thiago.fernando.lourenco@outlook.com

The research was not funded.

\section{ARTICLE INFO}

\section{Article history}

Received: April 25, 2018

Accepted: June 25, 2018

Published: July 31, 2018

Volume: 6 Issue: 3

Conflicts of interest: None

Funding: None

\begin{abstract}
Introduction: Knowing which physiological variables predict running performance could help coaches to optimize training prescription to improve running performance. Objective: The present study investigated which physiological respiratory responses could predict $3000-\mathrm{m}$ running performance. Methods: Seventeen amateur runners $(29.82 \pm 7.1$ years; $173.12 \pm 9.0 \mathrm{~cm} ; 64.59 \pm 9.3 \mathrm{~kg})$ performed a maximal graded running test on a treadmill. The ventilatory threshold (VT), respiratory compensation point (RCP), and maximal oxygen consumption (VO2max) were assessed, as well as the respective velocities (vVT, vRCP, vVO2max). After 72 to 96 hours the runners performed the 3000-m running field test. The relationships between variables were performed using Pearson product momentum correlations. Thereafter, simple and multiple regression models were applied. The significance level adopted was $5 \%(\mathrm{p}<0.05)$. Results: The majority of physiological responses were positive and well related to each other $(r \geq 0.70 ; p<0.05)$. Despite $v V T$, vRCP, and vVO2max demonstrating a higher and inverse relationship with 3000-m time $(r=-0.92 ; r=-0.96 ; r=-0.89 ; p<0.05)$, the multiple regression model indicated that $\mathrm{vRCP}$ and $\mathrm{vVO} 2 \mathrm{max}$ are the best variables to predict $3000-\mathrm{m}$ performance in experienced amateur road runners $(\mathrm{R} 2=0.94)$. The equation proposed by the model was: $3000-\mathrm{m}(\mathrm{s})=1399.21-[31.65 * \mathrm{vRCP}(\mathrm{km} . \mathrm{h}-1)]-\left[12.06 *_{\mathrm{vVO}} \mathrm{max}(\mathrm{km} . \mathrm{h}-1)\right]$. Conclusion: The $\mathrm{vRCP}$ and $\mathrm{vVO} 2$ max may be used to predict 3000 -m performance using only a maximal running test on a treadmill. In practical terms, coaches and physical conditioners can use performance in the 3000-m to select different exercise running intensities to prescribe exercise training intensities.
\end{abstract}

Key words: Running, Athletes, Exercise, Athletic Performance

\section{INTRODUCTION}

The number of road runners has increased greatly in recent years. In the USA, for example, there was an increase of $57 \%$ in road runners in the last decade. Moreover, there are estimated to be 50 million road runners in the USA and almost 14 million US road race participants in 2011 alone (Hryvniak, Dicharry, \& Wilder, 2014). The total number of US running events reached 26,370 in 2012. Similar events are held annually worldwide. For instance, the City to Surf in Sydney attracts 60,000 participants, and the Women's Mini Marathon in Dublin has over 40,000 participants, while the BUPA sponsored Great Run series attracts several hundred thousand participants across 15 events (Murphy, Lane, \& Bauman, 2015). In São Paulo, the most important economical Brazilian city (South America), from 2001 to 2015, road races increased from 11 to 415 with over 724,000 participants in 2015 . This represents an increase of $10.87 \%$ in comparison to 2014 (Atletismo, 2016). Finally, in 2016, the International Association of Athletics Federation (IAAF) included 88 road races worldwide in their official calendar (IAAF,
2017). Hence the trend in the number of competitions and practitioners looks likely to continue to increase in the near future. Due to the increase in road races worldwide, knowing the responses of physiological variables during some physical fitness tests, as well their relationship with simple, lowcost, fast, trustable, reproducible, easy, and valid field tests for running training prescription could help personal trainers, physical conditioners, coaches of amateurs, and sports scientists to prescribe better running training intensities and volumes, aiming to improve the physical fitness, pacing, wellbeing, health, and running performance of amateur runners (Maron, Douglas, Graham, Nishimura, \& Thompson, 2005; Starkoff, Eneli, Bonny, Hoffman, \& Devor, 2014).

Previous studies have reported that classic physiological respiratory variables such as those related to maximal aerobic power [i.e.; maximal oxygen uptake (VO2max), ventilatory threshold (VT), respiratory compensation point (RCP), and the velocity at which $\mathrm{VO} 2 \mathrm{max}$ occurs (vVO2max) present significant relationships with running performance (Arrese, Izquierdo, \& Serveto Galindo, 2006; Bellar \& Judge, 2012; Duffield, Bishop, \& Dawson, 2006; S, Craig, Wilson, \& 
Aitchison, 1997). Therefore, these variables have frequently been used to evaluate, prescribe, and monitor running training programs (Bragada et al., 2010; Bunc, Heller, Leso, Šprynarová, \& Zdanowicz, 1987; Esfarjani \& Laursen, 2007; Esteve-Lanao, Foster, Seiler, \& Lucia, 2007; Esteve-Lanao, Juan, Earnest, Foster, \& Lucia, 2005; Muñoz et al., 2014). However, the majority of these studies were conducted with elite or well-trained athletes and the relationship between these physiological variables and simplified field tests such as the 3000-m running test remain undescribed in amateur athletes. Therefore, the aim of the present study was to investigate the relationships between VT, RCP, VO2max, vVT, $\mathrm{vRCP}$, and $\mathrm{vVO} 2 \mathrm{max}$ assessed in a maximal running test on a treadmill until exhaustion and the 3000-m running field test. The hypothesis was that the running velocities related to these classic respiratory physiological responses would predict 3000-m running performance.

\section{MATERIAL AND METHODS}

The present work may be described as a descriptive cross-sectional study. All volunteers were informed of the procedures and signed the informed consent form. The procedures were conducted in accordance with the Helsinki Declaration for studies with humans, approved by the Ethics Committee of the Faculty of Medical Sciences of the State University of Campinas (n $\left.{ }^{\circ} 523 / 2010\right)$.

\section{Participants}

Seventeen amateur road runners (age: $29.82 \pm 7.10$ years; body height: $173.12 \pm 9.02 \mathrm{~cm}$; body weight: $64.59 \pm 9.39 \mathrm{~kg}$; BMI: $21.57 \pm 2.81$; training experience: $8.34 \pm 3.16$ years) participated in this study. All participants competed at regional level and were tested during their pre-competitive period. The volunteers were required to maintain their routines concerning fluid, food, sleep, physical activity, and recovery. At least 24-h before the tests, the volunteers were required to suspend their physical training.

\section{Maximal Graded Test}

The runners underwent a maximal incremental running test to determine the ventilatory threshold (VT), respiratory compensation point (RCP), and maximal oxygen uptake (VO2max). After a 3-min warm-up at $8-8.5 \mathrm{~km} \cdot \mathrm{h}^{-} 1$, the treadmill (Inbrasport Super-ATL, Porto Alegre, RS, Brazil) was set at $9 \mathrm{~km} \cdot \mathrm{h}^{-} 1$ and a fixed slope of $1 \%$ and after each 25 -sec interval, the running speed was increased by $0.3 \mathrm{~km} \cdot \mathrm{h}^{-} 1$ until volitional exhaustion according to Lourenço, Martins, Tesutti, Brenzikofer, \& Macedo (2011). Oxygen uptake (VO2), carbon dioxide output (VCO2), breathing frequency (Bf), and tidal volume $(\mathrm{Vt})$ were continuously collected with an automated breath-by-breath system (CPX/D Med Graphics, St. Paul, MN) using a Nafion filter tube and a turbine flow meter (opto-electric). Minute ventilation (Ve) was calculated as the product of Bf by VT, respectively. To analyze the data and decrease the variability in breath-by-breath acquisition we used the average of each 25 -s of exercise as recommend- ed by Robergs, Dwyer, \& Astorino (2010). Prior to each test, the analyzer was calibrated using a known gas mixture $(12 \% \mathrm{O} 2$ and $5 \% \mathrm{CO} 2)$, and the volume sensor was calibrated using a $3-\mathrm{L}$ syringe. The laboratory temperature was maintained at $21 \pm 1^{\circ} \mathrm{C}$ and the relative air humidity between $45-50 \%$. The VT and RCP were determined by non-invasive gas exchange measurements using the V-Slope method (Beaver, Wasserman, \& Whipp, 1986). The VT was detected by the loss of linearity of $\mathrm{VCO} 2$ as a function of $\mathrm{VO} 2$ during the incremental test. RCP was detected as the point of departure from linearity of the Ve vs. $\mathrm{VCO} 2$ relationship. The software supplied by Medical Graphics Breeze SuiteTM 6.4 MediGraphicsTM was used, supported by visual inspection from three independent and experienced researchers. The VO2max were considered as the value related to the last completed stage with a respiratory exchange ratio (RER) greater than 1.10 (Poole, Wilkerson, \& Jones, 2008).

\section{0-m Running Test}

From 72 to 96 hours after the maximal graded test the runners performed, individually, a 3000-m time trial on a 400-m outdoor track. The test began at 9 A.M. and after a standardized warm-up consisting of 15 minutes of jogging at a low speed (about 50\% of maximal 6-20 RPE scale), 10 to 15 minutes of active and ballistic stretching, and three 30 - to $50-\mathrm{m}$ sprints at increasing speeds, the athletes performed the $3000-\mathrm{m}$ running test. All participants were instructed to run as fast as possible at a self-selected pace and the total race time was measured using a stopwatch. The mean temperature was $24.2 \pm 2.2^{\circ} \mathrm{C}$ and the air humidity $47.4 \pm 1.8 \%$. The runners were allowed ad libitum hydration during the trial. Each subject was verbally encouraged to perform at maximum effort and could not use any kind of time device during the test.

\section{Statistical Analysis}

The normality of the data was checked using the Shapiro Wilk test. Data were described as mean $(\mathrm{X})$ and standard deviation (SD). Pearson product-moment correlation coefficients (r) and simple linear regression (R2) were used to determine the relationships between parameters. A multiple regression analysis (stepwise) was also used to evaluate the overall relationship between physiological (VT, RCP, VO2max), mechanical (vVT, vRCP, vVO2max), and 3000-m running test performance. The magnitudes of correlation $(90 \%$ confidence limits) between test measures were assessed as follows: $<0.1$, trivial $;<0.1-0.3$, small $;<0.3-0.5$, moderate $;<0.5-0.7$, large; $<0.7-0.9$, very large; and $<0.9-1.0$, almost perfect. If the 90 $\%$ confidence intervals overlapped small positive and negative values, the magnitude was deemed unclear; otherwise the magnitude was deemed to be the observed magnitude (Hopkins, Marshall, Batterham, \& Hanin, 2009).

\section{RESULTS}

The results of the maximal running test until exhaustion on the treadmill are described in Table 1. 
The results of the relationship between VT, RCP, VO2max, vVT, vRCP, vVO2max, and 3000-m are described in Table 2. The Pearson product-moment correlation coefficients $(\mathrm{r})$ and simple linear regression $\left(\mathrm{R}^{2}\right)$ showed very large $(\mathrm{r} \leq 0.7-0.9)$ and almost perfect $(\mathrm{r} \leq 0.9-1.0)$ correlations between all mechanical variables analyzed (vVT, vRCP, vVO2max) and 3000-m running test performance. The relationship between $\mathrm{vVT}$ and 3000-m running test performance was almost perfect $\left(\mathrm{r}=-0.92 ; \mathrm{R}^{2}=0.85 ; \mathrm{P}<0.05\right)$ as was found for $\mathrm{vRCP}$ and 3000 $\mathrm{m}$ running test performance $(\mathrm{r}=-0.96 ; \mathrm{R} 2=0.91 ; \mathrm{P}<0.05)$.

The linear regression to predict the $3000-\mathrm{m}$ running test performance is reported in Figure 1.

Table 1. Individual, average (X) and standard deviation (SD) of physiological responses and running velocity of a maximal running test until exhaustion on a treadmill and 3000-m time and velocity.

\begin{tabular}{|c|c|c|c|c|c|c|c|c|c|c|c|}
\hline \multirow[b]{2}{*}{ Athlete } & \multicolumn{3}{|c|}{ VT } & \multicolumn{3}{|c|}{ RCP } & \multicolumn{3}{|c|}{$\mathrm{VO}_{2} \max$} & \multicolumn{2}{|c|}{ 3000-m } \\
\hline & $\frac{\text { Vel }}{\left(\mathrm{km}^{\prime} \mathrm{h}^{-1}\right)}$ & $\begin{array}{c}\mathrm{VO}_{2} \\
(\text { L.min } \\
\left.\text {-1 }^{-1}\right)\end{array}$ & $\begin{array}{c}\mathrm{VO}_{2} \\
\left(\mathrm{ml}^{-\mathrm{kg}^{-1}} \cdot\right. \\
\left.\mathrm{min}^{-1}\right)\end{array}$ & $\frac{\text { Vel }}{\left(\mathrm{km}^{\left.-\mathrm{h}^{-1}\right)}\right.}$ & $\begin{array}{c}\mathrm{VO}_{2} \\
\left(\mathrm{~L} \cdot \mathrm{min}^{-1}\right)\end{array}$ & $\begin{array}{c}\mathrm{VO}_{2} \\
\left(\mathrm{ml}^{-k g^{-1}} \cdot\right. \\
\left.\mathrm{min}^{-1}\right)\end{array}$ & $\frac{\text { Vel }}{\left(\mathrm{km}^{\left.-\mathrm{h}^{-1}\right)}\right.}$ & $\begin{array}{c}\mathrm{VO}_{2} \\
\left(\mathrm{~L} \cdot \mathrm{min}^{-1}\right)\end{array}$ & $\begin{array}{c}\mathrm{VO}_{2} \\
\left(\mathrm{ml}^{-k g^{-1}} \cdot\right. \\
\left.\mathrm{min}^{-1}\right)\end{array}$ & $\begin{array}{c}\text { Time } \\
\text { (s) }\end{array}$ & $\begin{array}{c}\text { Vel } \\
\left(\mathrm{km}^{\prime} \mathrm{h}^{-1}\right)\end{array}$ \\
\hline 1 & 13.0 & 2.5 & 35.3 & 13.5 & 3.2 & 45.0 & 17.9 & 3.6 & 50.3 & 742.0 & 14.6 \\
\hline 2 & 12.1 & 2.3 & 35.4 & 13.0 & 1.7 & 34.4 & 17.9 & 2.0 & 42.1 & 763.0 & 14.2 \\
\hline 3 & 11.2 & 1.9 & 30.5 & 12.7 & 2.4 & 38.7 & 15.0 & 2.8 & 47.3 & 870.0 & 12.4 \\
\hline 4 & 13.8 & 2.5 & 35.3 & 16.4 & 3.2 & 45.0 & 22.2 & 3.6 & 55.9 & 619.0 & 17.4 \\
\hline 5 & 13.0 & 2.0 & 39.8 & 15.0 & 2.3 & 46.1 & 19.9 & 2.6 & 53.7 & 692.0 & 15.6 \\
\hline 6 & 14.7 & 2.5 & 31.4 & 17.3 & 2.5 & 35.4 & 22.8 & 3.6 & 55.6 & 606.0 & 17.8 \\
\hline 7 & 11.8 & 1.9 & 30.2 & 13.2 & 2.2 & 34.7 & 17.0 & 2.4 & 43.6 & 764.0 & 14.1 \\
\hline 8 & 11.5 & 1.7 & 36.0 & 12.7 & 1.9 & 38.6 & 15.6 & 2.0 & 42.1 & 814.0 & 13.3 \\
\hline 9 & 12.1 & 2.0 & 33.2 & 13.8 & 2.4 & 40.7 & 19.3 & 2.6 & 49.5 & 714.0 & 15.1 \\
\hline 10 & 11.8 & 2.4 & 29.9 & 13.0 & 2.5 & 32.2 & 15.8 & 3.1 & 39.6 & 770.0 & 14.0 \\
\hline 11 & 13.0 & 2.5 & 32.8 & 14.7 & 2.9 & 38.3 & 18.7 & 3.3 & 42.8 & 710.0 & 15.2 \\
\hline 12 & 13.2 & 1.8 & 28.4 & 15.0 & 2.2 & 34.8 & 18.4 & 2.6 & 40.0 & 721.0 & 15.0 \\
\hline 13 & 14.7 & 2.3 & 39.7 & 17.4 & 2.6 & 44.7 & 20.4 & 3.5 & 60.2 & 545.5 & 19.8 \\
\hline 14 & 17.7 & 2.4 & 40.0 & 19.2 & 2.5 & 42.2 & 21.9 & 3.2 & 54.4 & 537.3 & 20.1 \\
\hline 15 & 14.7 & 2.8 & 45.4 & 17.1 & 3.9 & 63.1 & 19.2 & 4.1 & 67.2 & 631.6 & 17.1 \\
\hline 16 & 15.9 & 1.7 & 30.4 & 17.7 & 2.1 & 37.5 & 19.2 & 2.6 & 46.4 & 596.7 & 18.1 \\
\hline 17 & 16.8 & 2.1 & 42.4 & 19.2 & 2.4 & 49.4 & 21.6 & 2.9 & 60.0 & 540.0 & 20.0 \\
\hline$X$ & 13.6 & 2.2 & 35.1 & 15.3 & 2.5 & 41.2 & 19.0 & 3.0 & 50.0 & 684.5 & 16.1 \\
\hline SD & 1.9 & 0.3 & 4.9 & 2.3 & 0.5 & 7.5 & 2.3 & 0.6 & 8.1 & 100.0 & 2.4 \\
\hline
\end{tabular}

Legend: VT - Ventilatory Threshold; RCP - Respiratory Compensation Point, $\mathrm{VO}_{2}$ max - Maximal Oxygen Consumption

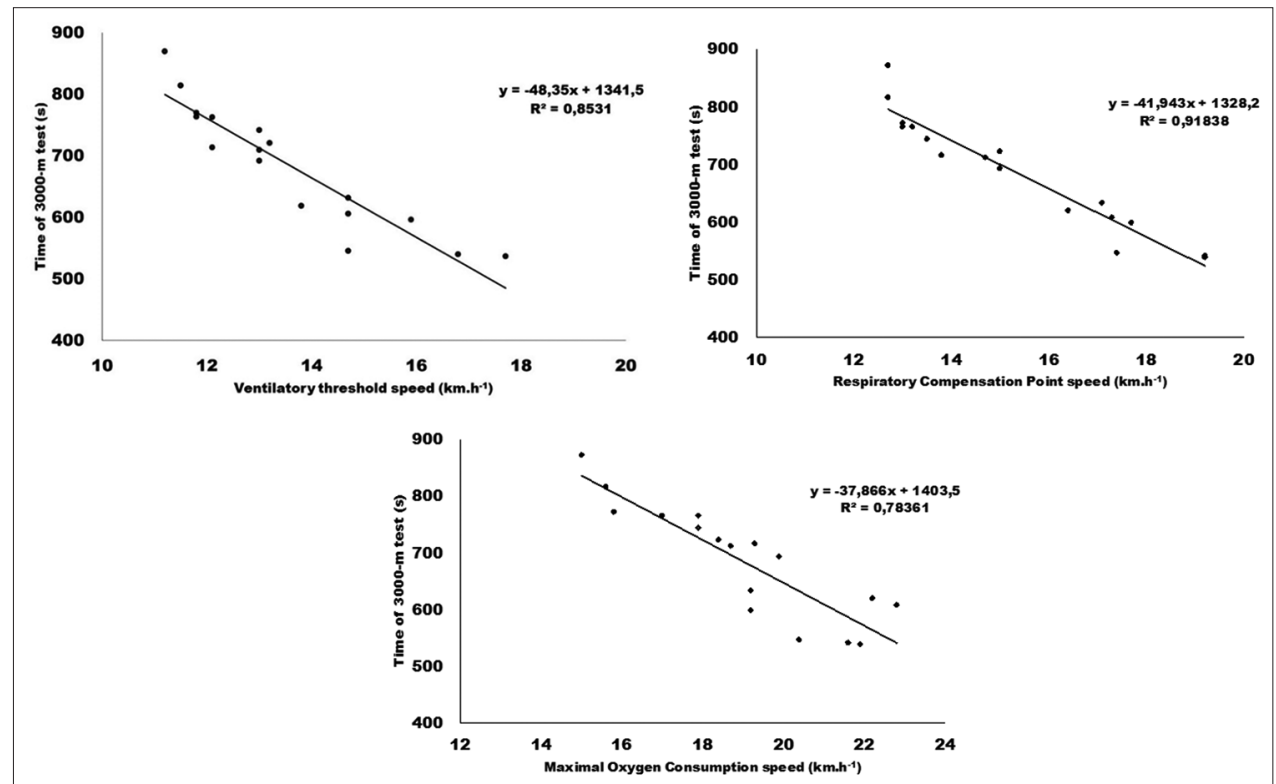

Figure 1. Linear regression of velocity of ventilatory threshold (Panel A), respiratory compensation point (Panel B), and maximal oxygen consumption (Panel C) to predict 3000-m running field test performance. 


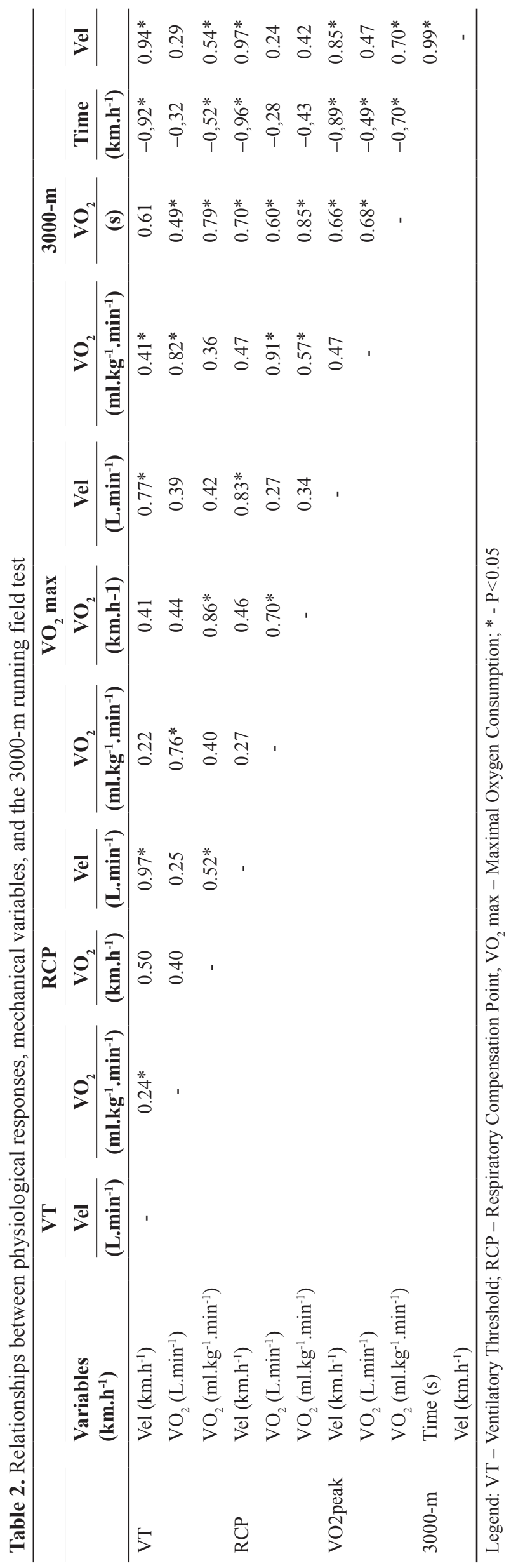

The multiple regression model is reported in Table 3.

The model indicates that the vRCP and vVO2max demonstrate better prediction of 3000-m running performance than the individual linear models $(\mathrm{F}=114.87 ; \mathrm{r}=$ $\left.0.97 ; \mathrm{R}^{2}=0.94 ; \mathrm{P}<0.05\right)$. The effect of $\mathrm{vVT}(\mathrm{P}>0.05)$ and ventilatory responses $\left(\mathrm{F}=0.07 ; \mathrm{r}=0.63 ; \mathrm{R}^{2}=0.40 ; \mathrm{P}>0.05\right)$ on 3000-m performance were not significant and were not included in the final model. The equation proposed by the model is described as follows:

$3000-\mathrm{m}(\mathrm{s})=1399.21-(31.65 * \mathrm{vRCP})-(12.06 *$ vVO2max)

(Equation 1)

\section{DISCUSSION}

The present study aimed to investigate the relationships between physiological respiratory responses of a graded maximal running test on a treadmill and 3000-m running field performance in amateur male runners. We hypothesized that running speeds related to the classic physiological responses such as vVT, vRCP, and vVO2max would demonstrate good to strong relationships to predict $3000-\mathrm{m}$ running performance. The main finding of the present study was that, as expected, the physiological variables were well related to each other. Furthermore, the multiple regressions showed that vRCP and vVO2max were the most important variables to predict 3000-m running performance. Although widely used in practice as an evaluative test, to our knowledge no work has investigated the relationship between 3000-m running performance and physiological responses in experienced amateur male runners. The results were according to our prior hypothesis since the Pearson product-moment correlation coefficients ( $\mathrm{r}$ ) and simple linear regression (R2) showed very large $(r \leq 0.7-0.9)$ and almost perfect $(\mathrm{r} \leq 0.9-1.0)$ correlations, with $\mathrm{vRCP}$ presenting the best relationship found $\left(\mathrm{r}=-0.96 ; \mathrm{R}^{2}=0.91 ; \mathrm{P}<0.05\right)$ followed by ${ }_{\mathrm{vVT}}\left(\mathrm{r}=0.94 ; \mathrm{R}^{2}=0.85 ; \mathrm{P}<0.05\right)$ and $\mathrm{vVO} 2 \mathrm{max}(\mathrm{r}=0.85$; $\left.\mathrm{R}^{2}=0.78 ; \mathrm{P}<0.05\right)$. These data are, in part, in agreement with those found by Yoshida et al. (1993), who found that $3000 \mathrm{~m}$ running performance of female athletes in distance runners $(10000 \mathrm{~m})$ was closely associated with blood variables such as lactate threshold $(\mathrm{r}=0.73)$, onset blood lactate accumulation $(\mathrm{r}=0.78)$, and VO2max $(\mathrm{r}=0.52)$. In addition, when performing the stepwise multiple regression, the authors also found that variables related to blood lactate contributed significantly to performance in the 3000-m. More recently, Bragada et al. (2010) showed similar results, indicating that $\mathrm{VVO} 2 \mathrm{max}$ and running speed at $4 \mathrm{mmol} \mathrm{L}^{-1}$ were strongly correlated to $3000-\mathrm{m}$ performance in welltrained middle-distance runners. However, in the present study, although we observed that the vVT, which corresponds to the lactate threshold, showed a higher correlation than these studies, when the stepwise multiple regression was performed it had no contribution to the 3000-m running performance. This may be due to the protocol used in other studies as whereas Bragada et al. (2010) used a fixed blood lactate concentration protocol which has been previously strongly questioned (see review of Faude, Kindermann, \& Meyer, 2009). Herein, we used a validated and reproducible protocol for the studied population (Lourenço et al., 2011). 
Table 3. Multiple regression model to predict 3000-m running test performance.

\begin{tabular}{lcccccc}
\hline Variable & Coefficient & Standard error & t-Stat & P-value & 95\% lower CI & 95\% upper CI \\
\hline Intersection & 1399.21 & 52.40 & 26.70 & 0.00 & 1286.82 & 1511.60 \\
vRCP $\left(\mathrm{km} \cdot \mathrm{h}^{-1}\right)$ & -31.65 & 5.08 & -6.22 & 0.00 & -42.55 & -20.74 \\
$\mathrm{vVO}_{2 \max }\left(\mathrm{km} \cdot \mathrm{h}^{-1}\right)$ & -12.06 & 4.97 & -2.43 & 0.03 & -22.72 & -1.41 \\
\hline
\end{tabular}

$\mathrm{vPCR}=$ respiratory compensation point velocity, $\mathrm{vVO}_{2}$ max $=$ maximal oxygen consumption velocity

Yoshida, Udo, Iwai, \& Yamaguchi (1993) used a protocol with higher stage times (5 minutes) to highlight the quantification of serum lactate concentration. Stage protocols lasting $>3$ minutes result in lower VO2max without consistency for vVT and vRCP determinations through gas analysis (Bentley, Newell, \& Bishop, 2007). It seems that smaller speed increments are more appropriate for determining vVT, vRCP, and vVO2max due to the mild adjustment of oxidative and glycolytic enzymes to compensate for the new adenosine triphosphate (ATP) demands (Bentley et al., 2007). Traditionally, lactate production/removal kinetics and ventilatory threshold are considered strong predictors of running performance ranging from $5-\mathrm{km}$ running to marathon (Faude et al., 2009; McLaughlin, Howley, Bassett, Thompson, \& Fitzhugh, 2010). This is in line with Billat, Binsse, Petit, \& Koralsztein (1998) and Lima-Silva et al. (2010) who considered that the ability to maintain running speed above VT represents one of the best predictors of 10$\mathrm{km}$ running performance. It is already known that exercises performed above vVT induce greater removal of lactate and hydrogen $\left(\mathrm{H}^{+}\right)$from the musculature, which may lead to a decrease in blood pH (Juel, 2008), reinforcing the importance of VRCP in the 3000-m performance, since RCP indicates the limit of blood buffering capacity (Wasserman, Beaver, Sun, \& Stringer, 2011). Furthermore, Noakes, Myburgh, \& Schall (1990) suggest that peak treadmill running velocity during the VO2max test alone predicts running performance. According to Billat, Renoux, Pinoteau, Petit, \& Koralsztein (1994) and Jones (1998), 3000-m running velocity ranged between 97 and 101\% vVO2peak (mean $100 \%$ ), which suggests that the $3000-\mathrm{m}$ race pace utilizes approximately $100 \%$ of the VO2peak. Instead, in the present study, we found that amateur runners performed the 3000-m at velocities located between vRCP and vVO2max, that is, at training intensity zone 3 . They performed at $105 \%$ of $\mathrm{VRCP}$ and at $84 \%$ of $\mathrm{VVO} 2 \mathrm{max}$, which reinforces the importance of plasma buffer capacity for endurance athletes. Theoretically, running speeds or exercise intensities above sRCP induce decreases in bpH and acidosis is detected by sensory feedback from working skeletal muscle, inducing hyperventilation to control blood pH (Amann et al., 2013; Bhambhani, Malik, \& Mookerjee, 2007). As a consequence, hyperventilation serves to reduce the arterial pressure of $\mathrm{CO}_{2}$ which has a direct effect on cerebral blood flow, and may decrease arterial oxygenation in the frontal cortex reducing/modifying the neural motor drive to protect the system and thereby choosing the exercise intensity slightly above vRCP (Amann et al., 2013; Bhambhani et al., 2007; Wasserman et al., 2011). Furthermore, as we found, the amateur runners performed the 3000-m at a slightly higher exercise intensity than $\mathrm{VRCP}$, suggesting the possibility of using the mean running speed of $3000-\mathrm{m}$ as a parameter to prescribe different exercise training intensities for this population. Although the data revealed high correlations between 3000$\mathrm{m}$ performance and a "gold standard" measure, the main limitation of the present study was the lack of control for wind speed, dehydration status, and convection load. It is already known that when running outdoors, air flows across the body at a speed equivalent to forward motion, which can aid heat loss through convection and evaporation and improve or impair running performance (Stevens \& Dascombe, 2015). Other studies should be designed to examine the influence of wind speed, dehydration, and convection load on the relationship between 3000-m and mechanical variables (vVT, vRCP, and vVO2max). Furthermore, we did not investigate any physiological parameters during the 3000-m time trial which opens the way for further studies to include these variables in their design. From a practical point of view, we can suggest that exercise intensities below the mean running speed of 3000-m would represent metabolic conditions similar to those found in zones 1 and 2 and, above this would represent zone 3 . These results can be useful to coaches and athletes as they involve a single exercise protocol, with minimal disturbance of the training routine and decreased recovery time, fatigue, and risk of injury, since they are amateur runners. Furthermore, the 3000$\mathrm{m}$ is a protocol that reproduces a situation close to the daily reality experienced by athletes.

\section{CONCLUSION}

We conclude that the vRCP and vVT showed the strongest relationship with 3000-m running performance. In practical terms, coaches and physical conditioners can use performance in the 3000-m to select different exercise running intensities which may be similar to those found in zone 1 (above vLT), zone 2 (between vVT and vRCP), and zone 3 (above vRCP).

\section{ACKNOWLEDGMENTS}

I thank all the athletes and coaches who participated in the study, highlighting the results found here.

\section{REFERENCES}

Amann, M., Venturelli, M., Ives, S. J., McDaniel, J., Layec, G., Rossman, M. J., \& Richardson, R. S. (2013). Peripheral fatigue limits endurance exercise via a sensory feedback-mediated reduction in spinal motoneuronal output. Journal of Applied Physiology, 115(3), 355-364. https://doi.org/10.1152/japplphysiol.00049.2013 
Arrese, A. L., Izquierdo, D. M., \& Serveto Galindo, J. (2006). Physiological measures associated with marathon running performance in high-level male and female homogeneous groups. International Journal of Sports Medicine, 27(4), 289-95.

Atletismo, F. P. de. (2016). Estatística 2016. Retrieved January 1,2017 , from http://www.atletismofpa.org.br/estatistica-2016.html,67

Beaver, W. L., Wasserman, K., \& Whipp, B. J. (1986). A new method for detecting anaerobic threshold by gas exchange. Journal of Applied Physiology, 60(6), 2020-7.

Bellar, D., \& Judge, L. (2012). Modeling and relationship of respiratory exchange ratio to athletic performance. Journal of Strength \& Conditioning and Research, 26(9), 2484-9.

Bentley, D. J., Newell, J., \& Bishop, D. (2007). Incremental exercise test design and analysis: implications for performance diagnostics in endurance athletes. Sports Medicine, 37(7), 575-86.

Bhambhani, Y., Malik, R., \& Mookerjee, S. (2007). Cerebral oxygenation declines at exercise intensities above the respiratory compensation threshold. Respiratory Physiology and Neurobiology, 156(2), 196-202. https://doi. org/10.1016/j.resp.2006.08.009

Billat, V., Binsse, V., Petit, B., \& Koralsztein, J. (1998). High level runners are able to maintain a VO2 steady-state below VO2peak in an all-out run over their critical velocity. Archieves of Physiology and Biochemistry, 3845(106), 1.

Billat, V., Renoux, J. C., Pinoteau, J., Petit, B., \& Koralsztein, J. P. (1994). Reproducibility of running time to exhaustion at VO2peak in subelite runners. Medicine and Science in Sports and Exercise, 26(2), 254-257.

Bragada, J., Santos, P. J., Maia, J. A., Colaço, P. J., Lopes, V. P., \& Barbosa, T. M. (2010). Longitudinal study in 3,000m male runners: relationship between performance and selected physiological parameters. Journal of Sports Science and Medicine, 9, 439-444.

Bunc, V., Heller, J., Leso, J., Šprynarová, Š., \& Zdanowicz, R. (1987). Ventilatory threshold in various groups of highly trained athletes. International Journal of Sports Medicine1, 8(4), 275-280.

Duffield, R., Bishop, D., \& Dawson, B. (2006). Comparison of the VO2 response to 800-m, 1500-m and 3000-m track running events. Journal of Sports Medicine and Physical Fitness, 46(3), 353-60.

Esfarjani, F., \& Laursen, P. B. (2007). Manipulating high-intensity interval training: Effects on, the lactate threshold and 3000-m running performance in moderately trained males. Journal of Science and Medicine in Sport, 10(1), 27-35.

Esteve-Lanao, J., Foster, C., Seiler, S., \& Lucia, A. (2007). Impact of training intensity distribution on performance in endurance athletes. The Journal of Strength \& Conditioning Research, 21(3), 943-949.

Esteve-Lanao, J., Juan, A. F. S., Earnest, C. P., Foster, C., \& Lucia, A. (2005). How do endurance runners actually train? Relationship with competition performance. Medicine \& Science in Sports \& Exercise, 496-504(37), 3.
Faude, O., Kindermann, W., \& Meyer, T. (2009). Lactate threshold concepts: how valid are they? Sports Medicine, 39(6), 469-90.

Hopkins, W., Marshall, S., Batterham, A., \& Hanin, J. (2009). Progressive statistics for studies in sports medicine and exercise science. Medicine \& Science in Sports \& Exercise, 41(1), 3-13.

Hryvniak, D., Dicharry, J., \& Wilder, R. (2014). Barefoot running survey: Evidence from the field. Journal of Sport and Health Science, 3(2), 131-136.

IAAF. (2017). Calendar 2017. Retrieved January 1, 2017, from https://www.iaaf.org/competition/calendar

Jones, A. M. (1998). A five year physiological case study of an Olympic runner. British Journal of Sports Medicine, 32(1), 39-43.

Juel, C. (2008). Regulation of pH in human skeletal muscle: Adaptations to physical activity. Acta Physiologica, 193(1), 17-24. https://doi.org/10.1111/j.17481716.2008.01840.x

Lima-Silva, A. E., Bertuzzi, R. C. M., Pires, F. O., Barros, R. V., Gagliardi, J. F., Hammond, J.,... Bishop, D. J. (2010). Effect of performance level on pacing strategy during a 10-km running race. European Journal of Applied Physiology, 108(5), 1045-1053. https://doi. org/10.1007/s00421-009-1300-6

Lourenço, T. F., Martins, L. E., Tesutti, L. S., Brenzikofer, R., \& Macedo, D. V. De. (2011). Reproducibility of an incremental treadmill $\mathrm{VO}(2)$ max test with gas exchange analysis for runners. Journal of Strength and Conditioning Research, 25(7), 1994-9.

Maron, B., Douglas, P., Graham, T., Nishimura, R., \& Thompson, P. (2005). Task Force 1: preparticipation screening and diagnosis of cardiovascular disease in athletes. J Am Coll Cardiol, 45, 1322-1326.

McLaughlin, J. E., Howley, E. T., Bassett, D. R., Thompson, D. L., \& Fitzhugh, E. C. (2010). Test of the classic model for predicting endurance running performance. Medicine \& Science in Sports \& Exercise, 42(5), 991-7.

Muñoz, I., Seiler, S., Bautista, J., España, J., Larumbe, E., \& Esteve-Lanao, J. (2014). Does polarized training improve performance in recreational runners? International Journal of Sports Physiology and Performance, 9(2), 265-272.

Murphy, N., Lane, A., \& Bauman, A. (2015). Leveraging mass participation events for sustainable health legacy. Leisure Studies, 34(6), 758-766.

Noakes, T. D., Myburgh, K. H., \& Schall, R. (1990). Peak treadmill running velocity during the VO2 max test predicts running performance. Journal of Sports Sciences, $8(1), 35-45$.

Poole, D. C., Wilkerson, D. P., \& Jones, A. M. (2008). Validity of criteria for establishing maximal $\mathrm{O} 2$ uptake during ramp exercise tests. European Journal of Applied Physiology, 102(4), 403-10.

Robergs, R. A., Dwyer, D., \& Astorino, T. (2010). Recommendations for improved data processing from expired gas analysis indirect calorimetry. Sports Medicine, 40(2), 95-111. 
S, G., Craig, I., Wilson, J., \& Aitchison, T. (1997). The relationship between $3 \mathrm{~km}$ running performance and selected physiological variables. Journal of Sports Sciences, 15(4), 403-410.

Starkoff, B. E., Eneli, I. U., Bonny, A. E., Hoffman, R. P., \& Devor, S. T. (2014). Estimated Aerobic Capacity Changes in Adolescents with Obesity Following High Intensity Interval Exercise. International Journal of Kinesiology \& Sports Science, 2(3), 1-8.

Stevens, C. J., \& Dascombe, B. J. (2015). The Reliability and Validity of Protocols for the Assessment of Endur- ance Sports Performance: An Updated Review. Measurement in Physical Education and Exercise Science, 19(4), 177-185.

Wasserman, K., Beaver, W. L., Sun, X. G., \& Stringer, W. W. (2011). Arterial $\mathrm{H}+$ regulation during exercise in humans. Respiratory Physiology and Neurobiology, 178(2), 191195. https://doi.org/10.1016/j.resp.2011.05.018

Yoshida, T., Udo, M., Iwai, K., \& Yamaguchi, T. (1993). Physiological characteristics related to endurance running performance in female distance runners. Journal of Sports Sciences, 11(1), 57-62. 